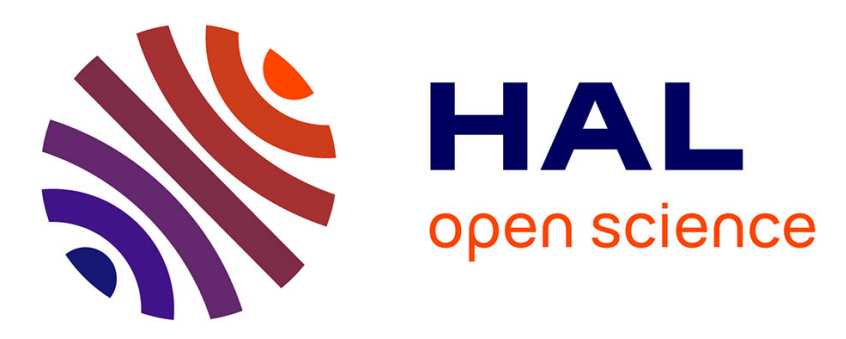

\title{
Spatial Frequency Reuse in a Novel Generation of PMR Networks
}

\author{
Alexis Lamiable, Joanna Tomasik
}

\section{To cite this version:}

Alexis Lamiable, Joanna Tomasik. Spatial Frequency Reuse in a Novel Generation of PMR Networks. 2013 IEEE Wireless Communications and Networking Conference (WCNC), Apr 2013, Shanghai, China. pp.1428-1433, 10.1109/WCNC.2013.6554770 . hal-00817794

\section{HAL Id: hal-00817794 \\ https://hal-centralesupelec.archives-ouvertes.fr/hal-00817794}

Submitted on 25 Apr 2013

HAL is a multi-disciplinary open access archive for the deposit and dissemination of scientific research documents, whether they are published or not. The documents may come from teaching and research institutions in France or abroad, or from public or private research centers.
L'archive ouverte pluridisciplinaire HAL, est destinée au dépôt et à la diffusion de documents scientifiques de niveau recherche, publiés ou non, émanant des établissements d'enseignement et de recherche français ou étrangers, des laboratoires publics ou privés. 


\title{
Spatial Frequency Reuse in a Novel Generation of PMR Networks
}

\author{
Alexis Lamiable and Joanna Tomasik \\ SUPELEC Systems Sciences (E3S), Computer Science Department \\ 3 rue Joliot-Curie, 91192 Gif-sur-Yvette Cedex, France \\ \{alexis.lamiable, joanna.tomasik\}@supelec.fr
}

\begin{abstract}
Private Mobile Radio (PMR) networks are cellular infrastructures dedicated to be used by professionals, such as public safety, military, industry and transportation organizations. In those networks, resources are scarce, and there are strong Quality of Service (QoS) requirements. The emergence of new services which need more bandwidth has made the world PMR leader focus on the LTE-Advanced protocol. In order to ensure the QoS despite resource shortage, we propose an algorithm of Resource Blocks (RBs) allocation with spatial frequency reuse whose scheme takes into account users' (UEs') interference possibility and probability.

We start by defining the underlying problem, which we call Weighted Fractional Coloring Problem (WFCP), in terms of graph theory. Next, we prove its NP-hardness. As obtaining an exact solution of such a problem in reasonable time is unrealistic, we propose a heuristic algorithm.

In order to evaluate the performance of our algorithm we use a rigorous validation procedure. We compare its performance with that of a random one which we propose as a reference and the exact one which can be run on very small networks. Thanks to the results obtained we believe that the proposed algorithm can establish a solid starting point to conceive its distributed versions for novel PMR protocols.
\end{abstract}

Index Terms-frequency reuse, resource allocation, optimization algorithms, interference control

\section{INTRODUCTION}

$\mathbf{P}$ RIVATE Mobile Radio (PMR) networks are cellular infrastructures dedicated to be used by professionals, such as public safety, military, industry and transportation organizations. In those networks, resources are scarce, notably on the up-link, and there are strong Quality of Service (QoS) requirements. The emergence of new services, which need more bandwidth, has made the world PMR leader Cassidian, a member of the EADS consortium, focus on enhancing of the LTE protocol for the next generation of PMR networks. The novel sensing mechanism considered by the PMR leader in future releases of its product will allow the interference possibility and probability of a user entity (UE) to be discovered more precisely than in the standard LTE networks. Knowing the UEs' interference interactions would enable us to allocate Resource Blocks (RBs) in a way that avoids intercell interference in a more flexible and efficient way than what is allowed by the frequency reuse schemes deployed nowadays [1].

We present what we call a spatial frequency reuse scheme, adapted to the uplink of the novel PMR architecture which we proposed within the context of the SOAPS.2 project. This project is also one of those of the SYSTEMATIC PARISREGION $^{1}$ competitiveness cluster.

We make several contributions in this paper. With the first one we get ahead in the modeling of problems of resource allocation with frequency reuse in the novel generation of PMR networks. We have at our disposal certain information about the UEs' probabilities of interfering with one another. This fact allows us to take advantage of these probabilities to reuse RBs in neighboring cells without creating interference.

We have been able to define this problem, which we call the Weighted Fractional Coloring Problem (WFCP), in a compact and elegant way, this opening up the possibilities of its theoretical analysis and conception of efficient algorithms to solve it (Section III). Therefore, despite its practical importance for the design of parsimonious and efficient spatial frequency reuse schemes, we are even more convinced that this problem is worth attention for purely theoretical reasons.

Our next contribution is the problem complexity computation. The NP-hardness result forces us to provide a heuristic algorithm, as exact methods cannot be considered for instances of relevant size. We propose a centralized algorithm based upon the greedy approach (Section IV) and the methodology of generation of random graphs necessary to validate it (Section V). As we deal with a newly stated problem, we have no other algorithms to compare with. For this reason, the algorithm performance evaluation is notably made under condition of a shortage of RBs (Section VI) in order to estimate the utilization of available resources. We also propose a centralized random allocation scheme as a performance reference. Another comparison is carried out against the exact method which is, however, feasible for very small networks due to its computational complexity. This confrontation gives us a clear idea of benefits in terms of transmission quality and RB utilization which can be made thanks to the new sensing technique.

Our paper starts with the state of the art in allocation schemes in standard LTE-based networks and coloring-based resource allocation models (Section II). The four following sections present our contributions and follow the order introduced in the paragraphs above. Section VII contains our conclusions and presents the perspectives arising from the WFCP formalization. It ends by the plan of our further work.

${ }^{1}$ SYSTEMATIC PARIS-REGION and the SOAPS.2 project are supported by the French Ministry of Industry, the department of Essonne, and the department of Yvelines. 


\section{RELATED WORKS}

RB allocation for the uplink has been studied notably in [2], [3], [4] and [5] and several algorithms have been proposed, both fast greedy algorithms and more time-consuming ones that are impractical for production use. These algorithms take the channel conditions into account, but do not avoid intercell interference. The usual assumption is that an appropriate frequency reuse scheme takes care of interference control.

Graph theory has been used before, notably in [6] and [7], to build an interference graph in which nodes model UEs, and two nodes are linked by an edge if the corresponding UEs are spatially close enough to interfere with each other. Graph coloring problems are used to represent the radio resource allocation problem, so that the same color (modelling an RB) is not attributed to adjacent nodes. However, the approach studied in [6] does not consider a possible preference of a node towards some specific colors, meaning any color is as good as another to color a given node. In practical applications, users have differing channel conditions, and not all RBs are equivalent.

The classical coloring heuristic DSATUR [8] is often used to color interference graphs, for example in [7] and [9]. In [7], DSATUR is used to obtain independent sets of nodes, and then channels are attributed to those sets depending on the channel conditions. The proposed algorithm is polynomial, in $\mathcal{O}\left(m n^{3}\right)$, where $m$ is the number of colors and $n$ the number of nodes.

\section{PROBlem Definition}

We use graph theory to define the optimal spatial frequency reuse scheme as a discrete optimization problem.

\section{A. Interference Graph}

Let us first define an interference graph as a graph $G=$ $(V, E)$ where nodes represent entities which can be assigned resources (for instance, UEs). Two nodes are linked by an edge in $E$ iff they are not to share the same resource because they would interfere with each other. In the specific case of SC-FDMA RB assignment, nodes $u$ and $v$ are linked in two cases:

- if UEs $u$ and $v$ are in the same cell, because of the frequency orthogonality constraint of SC-FDMA [10]

- if UEs $u$ and $v$ are not in the same cell, but $v$ is close enough to the eNodeB which handles $u$ so that it would create interference to the eNodeB if both UEs emitted on the same frequency

The latter point requires an interference probability map of the UEs, which will be made possible by a novel sensing mechanism for LTE-based PMR networks in development by Cassidian [1]. This sensing mechanism relies on the sharing between neighboring cells of the standard SRS (Sounding Reference Signal) mesurements, which will allow one to get an interference ability idea across multiple cells.

\section{B. Resource Blocks and Quality Metric}

In LTE-based systems, radio resources are allocated in the form of RBs. On the uplink, eNodeBs use SRS measurements sent by the UEs to assess, for these UEs, channel quality of all the available RBs. This measurement is used to build a scheduling metric. The scheduling metric can take into account other parameters, such as different priorities between UEs or a desire for fairness among UEs (for example, the classical Proportional Fair metric [11]). We will consider the chosen metric as a measure of quality.

To the interference graph $G=(V, E)$ above, we can therefore associate a quality matrix $M$ of size $|V| \times m$ if we have $m$ available RBs. Column $j$ of $M$ then corresponds to a vector containing the nodes' preferences towards color $j$. Our objective is, on the one hand, to find an assignment of RBs to UEs that does not attribute the same RB to adjacent UEs in the interference graph, which is a graph fractional coloring problem [12]. On the other hand, we want to maximize the overall transmission quality, as defined by matrix $M$ (we consider $M_{i j}$ as the weight of color $j$ for node $i$ ). We model this by a new problem that we call the Weighted Fractional Coloring Problem (WFCP).

\section{Weighted Fractional Coloring Problem}

Let $G$ be an interference graph, and let $m$ colors (corresponding to RBs) be available. We want to find a coloring of graph $G$ which does not attribute the same color to two adjacent nodes. We are allowed to use up to $m$ colors for a single node, but some nodes may remain uncolored. Our objective is to find such a coloring that also maximizes the sum of the weights of the colors attributed to nodes.

It should be noted that, as we are considering the uplink, UEs have power constraints. An UE has to divide its power over all the RBs it emits on. Therefore, we introduce a demand $d$ that is fixed for all nodes and that represents the maximum number of RBs a node demands to be allocated.

\section{Definition 1. Weighted Fractional Coloring Problem (WFCP)}

Instance: $G=(V, E)$ an undirected graph, a mapping $w(v, j)$ of nodes $v$ and RBs $j$ to natural numbers (weights), a demand $d, d \in \mathbb{N}$.

Objective: find for all nodes $v$ a set $A_{v}=\{j: 1 \leq j \leq m\}$ (possibly empty) such that:

- $(u, v) \in E$ implies $A_{u} \cap A_{v}=\emptyset$

- $\forall v \in V,\left|A_{v}\right| \leq d$

- $\sum_{v \in V} \sum_{j \in A_{v}} w(v, j)$ is maximum.

Theorem 1. The WFCP is NP-hard.

Proof: Let us consider the special case where $d=m=1$. Coloring the graph with a single color $c$ is equivalent to finding an independent set of nodes in that graph (i.e. a subset $V^{\prime} \subseteq V$ such that no two nodes in $V^{\prime}$ are joined by an edge in $E$ ). The quality vector related to color $c$ corresponds to adding weights to the nodes, and trying to find an independent set of maximum total weight. We recall the definition of the Weighted Independent Set decision and optimization problems.

Definition 2. Weighted Independent Set Problem (WISP) [13] Instance: $G=(V, E)$ an undirected graph, a mapping $w$ of 
nodes to natural numbers (weights $w: V \rightarrow \mathbb{N}$ ), and $k$ a natural number.

Question: does $G$ contain an independent set whose weight is greater than or equal to $k$ ?

This problem is NP-complete. In fact, even its special case where $\forall v \in V, w(v)=1$ is NP-complete [13]. Hence, the associated maximization problem, as defined below, is NPhard.

Definition 3. Maximum Weighted Independent Set Problem (MWISP)

Instance: $G=(V, E)$ an undirected graph, a mapping $w$ of nodes to natural numbers (weights $w: V \rightarrow \mathbb{N}$ ).

Objective: find an independent set of maximum weight.

The MWISP problem is a special case of the WFCP and is NP-hard. Therefore, the WFCP is also NP-hard.

Since the WFCP is NP-hard, exact solutions will only be available for extremely simple instances. We therefore propose a heuristic algorithm.

\section{Granularity}

As defined above, and as used throughout this paper, nodes represent UEs. However, different granularity levels can be used. In the most coarse-grain model, nodes represent cells, neighboring cells should not share RBs, and we are in the classical Fractional Frequency Reuse scheme. In the most finegrain model, presented here, we attribute RBs to users directly. But in intermediate models, groups of neighboring users could be considered as nodes. This could reduce the size of the graph significantly at only a slight performance cost, and is particularly relevant in a PMR application where users could be grouped by patrol or by event.

\section{HeuristiC Algorithm}

Algorithm 1 describes our heuristic, greedy algorithm. We basically build a list of all available colors for all the nodes, find the pair with highest metric, assign it, remove all nowunavailable colors from the list, and start again. Line 8 is an example of how we can adjust the metric during the run of the algorithm, in order to avoid having the best located clients pumping out all the resources. It can be omitted or adapted to fit different QoS requirements.

The while loop will run at most $m n$ times; all operations inside it are in $\mathcal{O}(m n)$. Consequently, the algorithm is in $\mathcal{O}\left(m^{2} n^{2}\right)$ in the worst case.

\section{INTERFERENCE GRAPH GENERATION}

We validated our heuristic algorithm solving the WFCP on series of random interference graphs in order to estimate its performance. In order to do this, we needed a way to produce series of random interference graphs that were plausible.

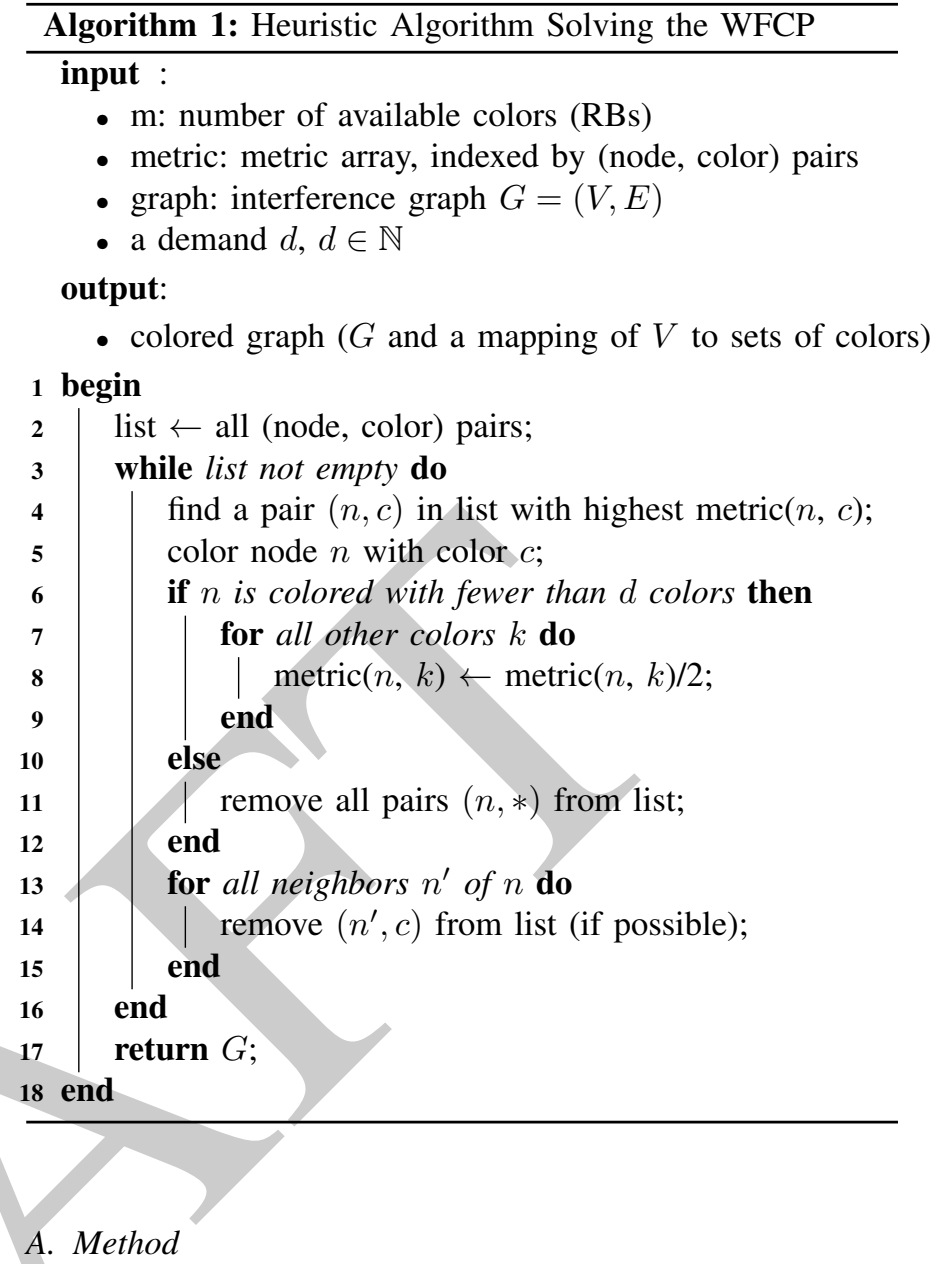

Our idea to obtain interference graphs was to generate random topologies of a fixed size according to commonly approved models and "span" them on PMR cells. We opted for the widely used Waxman model [14] and the extended Barabási and Albert model [15]. The first one is typically used to represent a uniform graph with given numbers of nodes and edges. We chose it to model a "non-emergency" situation (UEs dispersed regularly). The second one expresses the property of power law distribution of the node degree which may often be observed in man-made communication networks. We selected it to model an emergency situation (certain UEs' concentrations). We use aSHIIP [16] for graph generation, our home-made and publicly available tool ${ }^{2}$, which offers a powerful and reliable topology generator. We spanned generated topologies of 70 UEs on a standard honeycomb made up of seven cells. The spanning procedure is composed of four steps.

First, we identified two methods to set the cell centers (eNodeBs' localization) in order to attribute UEs to cells. Under our assumption a selected UE stands close to an eNodeB in each cell. According to the first method we select seven centers in a uniform way. The second method takes into account properties of a topology and sets the centers homogeneously. In other words, the cell centers are a solution to the $k$-center problem ( $k=7$ in our experiments) which

\footnotetext{
${ }^{2}$ http://wwwdi.supelec.fr/software/ashiip/
} 
is NP-complete [13]. We find the centers with the heuristic algorithm proposed in [17].

As a reader may already have noticed there is always a UE very close to an eNodeB. After being switched on it has a tendency to "pump out" available resources in a cell as its metric is, most probably, the highest in this cell. In eBAbased topologies with homogeneous eNodeB placement this tendency is stronger because the $k$-center algorithm designates as centers the nodes whose degree is relatively great.

Second, we associate nodes to cells. We express the distance between a node and a cell center as an edge count. The nodes closer to a cell center are associated to the corresponding cell. In the case in which a node is equally far from several centers, the decision is taken by a uniform draw.

Third, we designate the central honeycomb cell as the one which is the most strongly connected to the other cells. The first neighboring cell is the one the most strongly connected (in terms of the edge number) with the central one. The second neighbor cell is the one the most strongly connected to the first neighbor, etc. The last, sixth neighbor of the central cell is finally connected to the first neighbor.

Finally, a honeycomb spanned topology is cleaned up by removing edges between cells which are not neighbors and cell subgraphs are considered to be complete. These cliques model the fact that the same RB cannot be assigned to several UEs within the same cell (i.e. the orthogonality constraint from SD-FDMA). An example of topology derived from the eBA model and with the homogeneous cell centers' placement used in the performance evaluation procedure is illustrated in Fig. 1. Two topology generation models and two methods of cell center setting give us interference graphs in cases of non-emergency/emergency UEs deployment and uniform/homogeneous eNodeB placement.

\section{B. Parameters}

We analyzed series of 1000 topologies of each type in order to observe their characteristics. We calibrated the initial generation models (Waxman $\alpha=0.2, \beta=0.25$, eBA $m 0=10, m=1, p=0.5, q=0.2$ ) to keep the mean size of cells comparable. Indeed, the confidence intervals for these means on 0.05 confidence level are $(11.21,11.27)$ and $(10.61,10.71)$, respectively. At the same time the mean size of entire topologies is about of 69 nodes for both models as the spanning procedure may result in several nodes whose metric value is so weak that they cannot be connected. We observed that interference graphs derived from the Waxman model are statistically identical despite a spanning procedure. As eBA topologies are denser than those obtained with the Waxman model, the mean node degree in eBA-based interference graphs is approximately twice as high as in Waxman-based ones.

Series of interference graphs needed for pertinent validation have to have the assignment of metric values to available RBs for each UE done in a coherent way. We opt for a normal distribution rescaled in function of the distance between a UE and an eNodeB expressed as a number of edges:

$$
\operatorname{metric}(\mathrm{UE})=\frac{N(\mu, x \mu)}{\operatorname{dist}(\mathrm{UE}, \mathrm{eNodeB})+1} .
$$

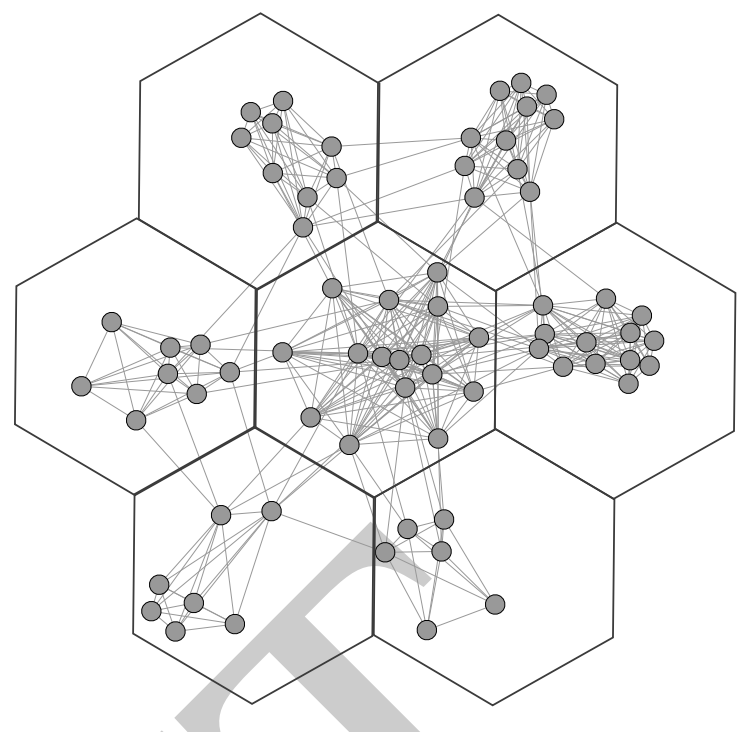

Fig. 1. An example of an eBA generated interference graph with homogeneous eNodeB placement, spanned on the honeycomb

This linear formula preserves metric values from decaying too quickly and makes graphs "harder to be solved" by reuse schemes. We point out that edges added to form complete graphs out of sub-graphs representing cells are not counted in our formula. We argue here that an edge in graphs in our experiences defines the existence of interference, not a geographical distance and we are not attempting to violate any law concerning signal attenuation. We put arbitrarirly $\mu=50$ and $x=15 \%$ in Eq. (1).

An interference graph of size $n$ (whose nodes correspond to UEs), $m$ available colors (corresponding to RBs), a demand of $d$ RBs per UE, and metric values (corresponding to color weights) constitute an instance of the WFCP.

\section{Vi. Performance Evaluation and Results}

We compare the WFCP algorithm performance with those of a random one, which we propose in Subsection VI-A, on series of 1000 random interference graphs for all four scenarii.

The algorithms realize frequency allocation only; metric values do not depend therefore on allocations done in the past. In other words, the frequency attribution is made at time $t$ when all UEs have just switched on and want to emit. We chose the average transmission quality and number of allocated RBs as performance measures.

Then, in Subsection VI-B, we attempt to compare solutions of our heuristic algorithm with exact ones. Due to the WFCP complexity, we can carry on this examination for very small interference graphs.

\section{A. Random Algorithm}

Algorithm 2 is similar to the heuristic algorithm but does not consider the metric when choosing which (node, color) pair to pick, line 4. Instead, pairs are chosen with uniform probability from the list.

The experiments for the series of 1000 random graphs constructed according to the description in Subsection V with 

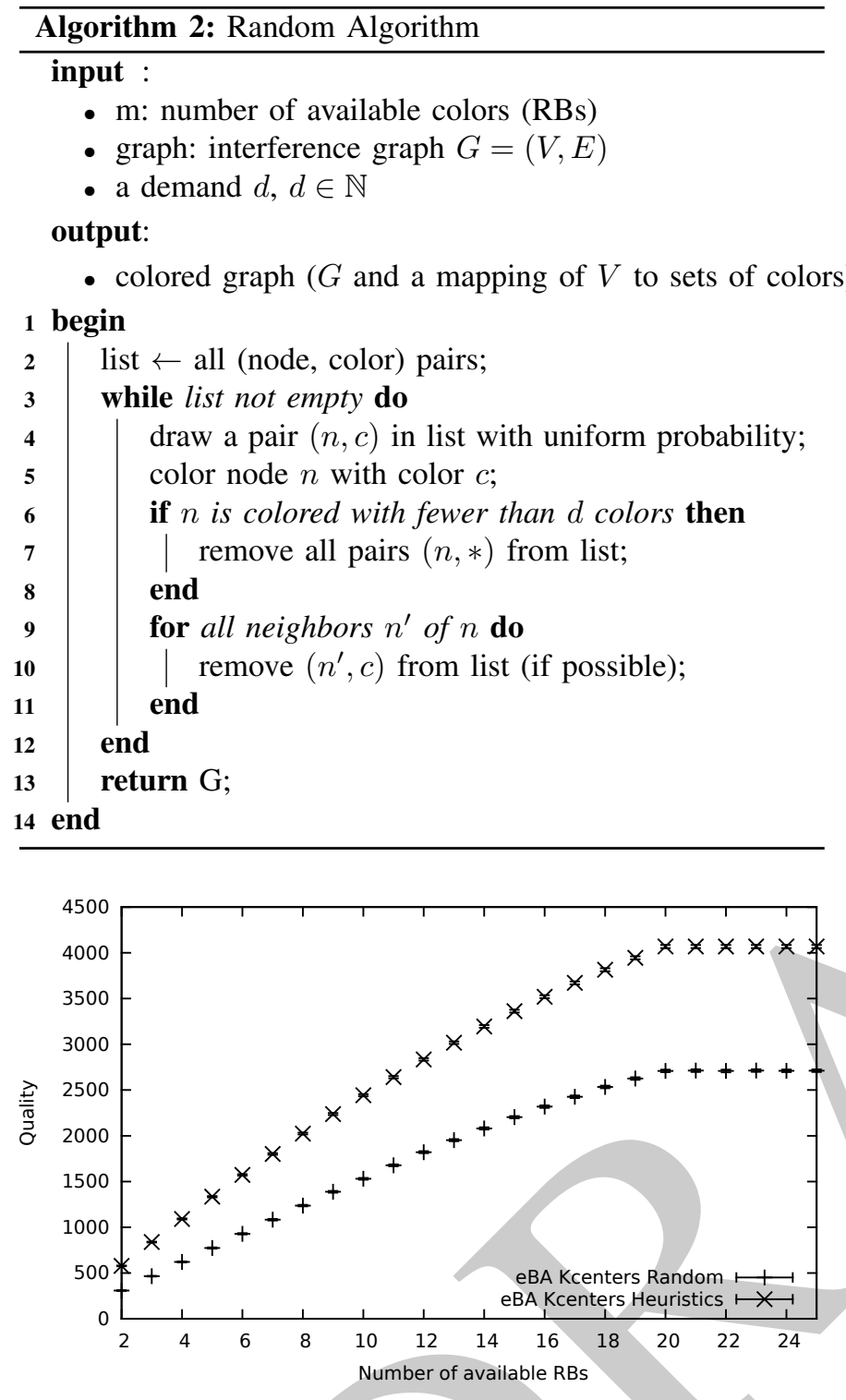

Fig. 2. Results for graphs with eBA topology and K-center cell distribution.

$d=2$ and varied $m$. As the average cell size is of 10 UEs, the availability of $m=20 \mathrm{RBs}$ is sufficient to satisfy the demands of all the UEs. We start by computing the average signal quality for transmission for four scenarii identified in Subsection V in function of the number $m$ of available RBs. These averages, together with their confidence intervals with $\alpha=0.05$, which are extremely tight, are depicted in Fig. 2 for the eBA topology model and the $k$-center based cell distribution. As expected, our algorithm clearly performs better than the random one. The other three scenarii produce similar results and are not shown here in detail.

The ratio of signal quality obtained with the heuristic and random algorithms in function of the number of available RBs is illustrated in Fig. 3. When RBs are satisfactorily numerous to fulfill the global demand, $m \geq 20$, the WFCP heuristic algorithm outperforms the random one by about 50\% despite the interference graph type. This difference is even more evident in the case of resource shortage. For instance, for

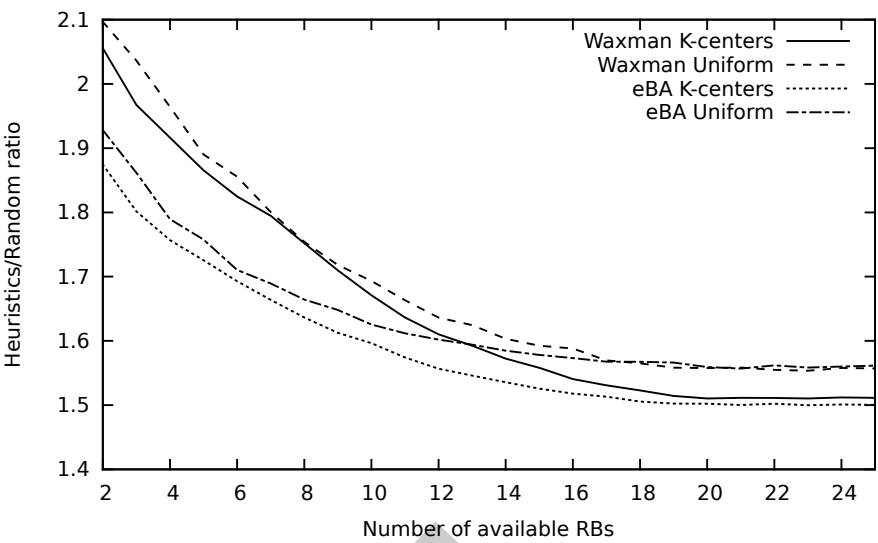

Fig. 3. Ratio between the heuristic and random solutions (signal quality) as a function of the number of available RBs.

$m=4$, the random algorithm performs almost half as well as the heuristic one for all the scenarii.

In the same figure we observe that this ratio is greater for Waxman-based graphs. This difference can be explained by the fact that the heuristic algorithm's "worst case" graph is a star graph where the center node has weight $w_{1}$ and the other $k$ nodes have weight $w_{2}$, with $k w_{2}>w_{1}$. Graphs with an eBAbased topology are more likely to contain such situations than Waxman-based graphs. This is even more problematic with a $k$-center based cell distribution. In those graphs, the strongly connected nodes are very likely to be solutions to the $k$-center problem, which will make them cell centers, and as such will give them higher weights. As shown in Fig. 3, those graphs do have the lowest ratio.

The influence of eNodeBs' localization is much less distinct and it may be observed principally in the situation of resource abundance $(m \geq 20)$ in Fig. 3. For the same reason as that explained below, the random algorithm may happen to choose randomly a node placed close to an eNodeB and this choice may be advantageous. Consequently, the surplus of RBs makes the algorithms "topology insensitive" and the dominant factor of their performance is the eNodeB placement.

\section{B. Exact Algorithm and "Toy Instances"}

The exact method to solve the WFCP has the complexity in $\mathcal{O}\left((m+1)^{n d}\right)$. It can thus be performed in reasonable time only for small instances which are without any practical interest. We compute, however, the exact results for toy examples to confront them with those obtained with our heuristic algorithm.

Interference graphs the size of 15 UEs are deployed on the same seven cell honeycomb as random ones (Fig. 4(a)). The central cell is a clique of three UEs, its six neighbors are cliques of two UEs. The central cell is connected to its neighbors either by one link or two links, alternately. As we cannot really determine the eNodeBs' localization in such a trivial structure, we attribute metric values within a cell according to $u N(\mu, x \mu)$, where $u$ is uniform on $(0,1)$. For computational reasons we have to limit ourselves to $m=2$, and $d=2$. With these parameters, our heuristic algorithm 

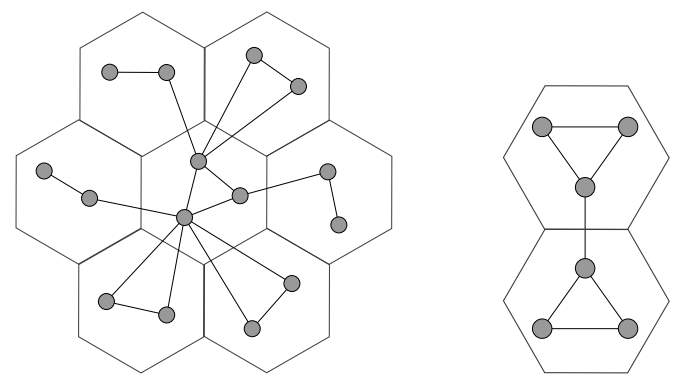

(a) Toy instance with 15 nodes (b) "Apple core" graph, seen as spanned on the seven cells hon- a two cell network. eycomb.

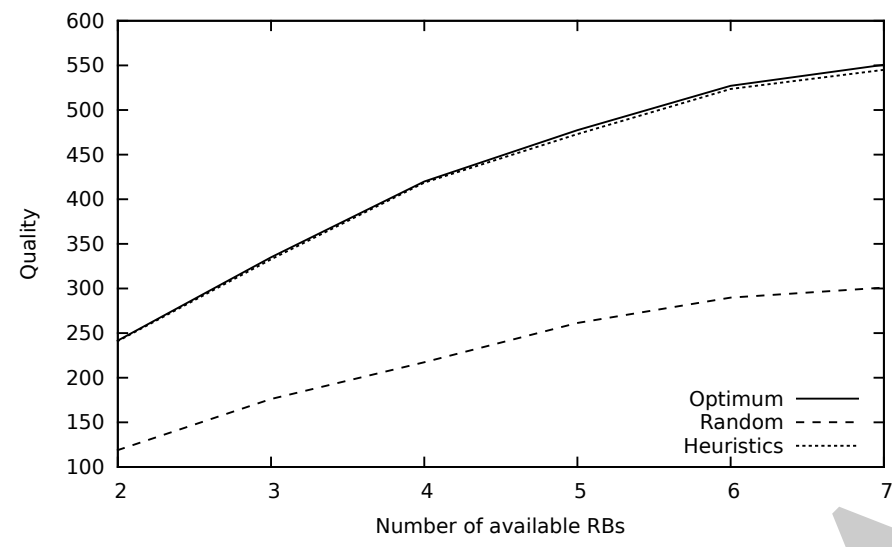

Fig. 4. Comparison between the performances on the apple-core graph of the random and the heuristic algorithms and the optimal result, depending on the number of available RBs.

found the exact solution in $75 \%$ of the randomly generated metric matrices. When it did not, the computed solution was at worst $94 \%$ of the optimal one.

In order to bring together the heuristic and exact methods' results in function of a varied number of available RBs we must restrain our ambitions regarding the size of the interference graph even more. We propose for the further performance analysis an "apple-core" graph, Fig. 4(b).

With $d=2$, this toy network needs $m \geq 12$ RBs to satisfy its demands entirely. For computational reasons we cannot reach this limit. The average signal quality computed with the heuristic, random and exact algorithms is depicted in Fig. 4. All three cases are averaged over 20 random metric matrices, and results for the random algorithm are averaged over 50 runs for each matrix. For an apple-core-like graph and for a given $m$, the heuristic algorithm is two or three times better than the random one. Moreover, the results of the heuristic one are statistically the same as those obtained in the exact way. This is an encouraging observation despite the small instance size.

\section{CONCLUSION AND FURTHER WORK}

The passage towards broadband transmissions in LTE-based PMR networks makes Cassidian, the world leader in this field, propose new functionalities of this protocol. This new feature concerns a more precise sensing mechanism allowing a more efficient spatial reuse of available resources and, accordingly, improves the level of the UEs' QoS satisfaction, which is crucial in this type of professional networks.

Our first goal was to define a problem which models the attribution of frequencies to cells with maximization of the transmission quality having at our disposal UEs' interaction probabilities obtained by the novel sensing mechanism. Our algorithmic approach authorizes the same RB to be allocated in neighboring cells without creating interference and, consequently, improve the frequency reuse. After defining the WFCP we analyzed it from a theoretical point of view.

As the WFCP is NP-hard, we proposed a heuristic algorithm of low complexity to solve it and confronted its performance with those of a random one and of the exact one, the latter being feasible for networks with very few UEs only.

In this paper we have built the solid fundaments for the spatial frequency reuse scheme for the new generation of PMR networks. The results of our heuristic algorithm, which we have presented herein, encourage us to pursue the development of its distributed versions keeping in mind their possible implementation in future PMR protocols. The next natural step will be the introduction of the scheduling of UEs' demands in time taking into account namely UEs' priorities, traffic prevision, and degradation of a transmission range in function of the number of RBs emitted in the same slot. To validate these approaches we will use the introduced methodology of random generation of interference graphs. As we have announced in Subsection III-D, we will also work on this model at different granularity levels.

\section{REFERENCES}

[1] Partners of SOAPS.2 project, "Self-adaptive frequency resource allocation," Cassidian, Tech. Rep., 2012, http: //soaps2.fr.

[2] F. D. Calabrese, "Scheduling and Link Adaptation for Uplink SC-FDMA Systems," Ph.D. dissertation, Aalborg University, April 2009.

[3] M. Anas, "Uplink Radio Resource Management for QoS Provisioning in Long Term Evolution With Emphasis on Admission Control and Handover," Ph.D. dissertation, Aalborg University, January 2009.

[4] F. I. Sokmen and T. Girici, "Uplink Resource Allocation Algorithms for Single-Carrier FDMA Systems," Proc. of EWC, 2010.

[5] L. A. M. R. de Temiño, G. Berardinelli, S. Frattasi, and P. Mogensen, "Channel-Aware Scheduling Algorithms for SC-FDMA in LTE Uplink," Proc. of PIMRC, 2008.

[6] M. C. Necker, "A graph-based scheme for distributed interference coordination in cellular OFDMA networks," in Proc. of VTC, 2008.

[7] M. Pischella and J. C. Belfiore, "Graph-based weighted sum throughput maximization in OFDMA cellular networks," in Proc. of IWCLD, 2009.

[8] D. Brélaz, "New methods to color the vertices of a graph," Communications of the ACM, vol. 22, no. 4, 1979.

[9] S. Pomportes, A. Busson, J. Tomasik, and V. Vèque, "Resource allocation in ad hoc networks with two-hop interference resolution," in Proc. of. GLOBECOM, 2011.

[10] H. G. Myung, J. Lim, and D. J. Goodman, "Single carrier FDMA for uplink wireless transmission," Vehicular Tech Mag, vol. 1, no. 3, 2006.

[11] H. Kim, K. Kim, Y. Han, and S. Yun, "A proportional fair scheduling for multicarrier transmission systems," in Proc. of VTC, vol. 1, 2004.

[12] R. Edward and D. Ullman, "Fractional graph theory," 1997.

[13] M. R. Garey and D. S. Johnson, Computers and intractability: A guide to the theory of NP-completeness. Freeman, 1979.

[14] B. M. Waxman, "Routing of multipoint connections," JSAC, vol. 6, no. 9, 1988.

[15] R. Albert and A.-L. Barabási, "Topology of evolving networks: Local events and universality," Physical Review Letters, vol. 85, 2000.

[16] J. Tomasik and M.-A. Weisser, "aSHIIP: autonomous generator of random Internet-like topologies with inter-domain hierarchy," in Proc. of MASCOTS, 2010.

[17] J. Mihelic and B. Robic, "Solving the k-center problem efficiently with a dominating set algorithm," CIT, vol. 13, no. 3, pp. 225-234, 2005. 